Binghamton University

The Open Repository @ Binghamton (The ORB)

4-28-2019

\title{
A parametric resonator with low threshold excitation for vibration energy harvesting
}

Shahrzad Towfighian

Binghamton University--SUNY, stowfigh@binghamton.edu

Wei Yang

Binghamton University--SUNY, wyang24@binghamton.edu

Follow this and additional works at: https://orb.binghamton.edu/mechanical_fac

Part of the Mechanical Engineering Commons

\section{Recommended Citation}

W. Yang, S. Towfighian (2019), A Parametric Resonator with Low Threshold Excitation for Vibration Energy Harvesting, Journal of Sound and Vibration, Vol. 446, pp. 129-143.

This Article is brought to you for free and open access by the Mechanical Engineering at The Open Repository @ Binghamton (The ORB). It has been accepted for inclusion in Mechanical Engineering Faculty Scholarship by an authorized administrator of The Open Repository @ Binghamton (The ORB). For more information, please contact ORB@binghamton.edu. 


\title{
A parametric resonator with low threshold excitation for vibration energy harvesting
}

\author{
Wei Yang ${ }^{\mathrm{a}}$, Shahrzad Towfighian ${ }^{\mathrm{a}, *}$ \\ ${ }^{a}$ State University of New York at Binghamton, Binghamton, New York 13902
}

\begin{abstract}
A parametric resonator for vibration energy harvesting is presented. Despite large responses from parametric resonance, two major drawbacks of parametric resonance harvesters are the high threshold excitation and narrow bandwidth. We addressed these two shortcomings by adding magnetic nonlinearity to the system. The proposed vibration energy harvester consists of two piezoelectric cantilevers beams, each with a magnetic tip. By controlling the distance between the two magnets, the threshold excitation level needed to trigger the parametric resonance decreases. Combining the softening and hardening behavior of the two magnetically coupled beams increases the frequency bandwidth. In addition, the amplitude of the response increases with the merger of the direct and parametric resonances of the two beams. We present a mathematical model of the system consisting of two lumped systems coupled by the magnetic force. The coupled governing equations are solved numerically, analytically, and are verified by experiments. Unique characteristics of wider bandwidth, larger response, and lower threshold excitation occur at the low frequency because of the added magnetic nonlinearity to the two-beam system. These properties can improve the efficiency of vibration energy harvesters.
\end{abstract}

Keywords: Nonlinear vibration, energy harvesting, parametric resonance, piezoelectric

\footnotetext{
*Corresponding author

Email addresses: wyang24@binghamton.edu (Wei Yang), stowfigh@binghamton.edu (Shahrzad Towfighian)
} 


\section{Nomenclature}
$X: \quad$ Deflection of horizontal beam
$Y: \quad$ Deflection of vertical beam
$m_{1}$ : $\quad$ Effective mass of horizontal beam and magnet
$m_{2}$ : $\quad$ Effective mass of vertical beam and magnet
$k_{1}: \quad \quad$ Stiffness of horizontal beam
$k_{2}$ : $\quad$ Stiffness of vertical beam
$c_{1}$ : $\quad$ Equivalent viscous damping of horizontal beam
$c_{2}: \quad$ Equivalent viscous damping of vertical beam
$d$ : $\quad$ Initial distance between magnets (center to center)
D : $\quad$ Total distance between magnets (center to center)
$x$ : $\quad$ Dynamic response of vertical beam
y : Dynamic response of horizontal beam
$t: \quad$ Time
$x_{b}: \quad$ Base excitation
$c_{p}: \quad$ Capacitance
$R: \quad \quad$ Load resistance
$\theta_{e}: \quad$ Coupling coefficient
$M: \quad$ Magnetization moment of magnet
$\mu_{0}: \quad$ Permeability of space
$v_{1}$ : $\quad$ Output voltage from the horizontal beam
$v_{2}: \quad$ Output voltage from the vertical beam
$\sigma, \sigma_{1}, \sigma_{2}:$ Small detuning parameters
$T_{0}, T_{1}$ : Two time scales
$\varepsilon: \quad$ Scaling parameter
$y_{c}: \quad$ Deflection of the beam in conventional bistable system
$\mu_{c}: \quad$ Equivalent damping ratio in conventional bistable system
$\Omega: \quad$ Excitation frequency
$A_{a}: \quad$ Base excitation in conventional bistable system
$\omega_{n c}: \quad \quad \quad$ Natural frequency of the beam in conventional bistable system
$\alpha: \quad \quad$ Cubic nonlinearity in conventional bistable system
$k: \quad \quad \quad$ Effective stiffness of the beam and magnetic tip in conventional bistable system
$D_{c}: \quad$ Total distance between magnets in conventional bistable system

\section{Introduction}

Energy harvesting systems have become a prominent research area in the last two decades. Applications include self-powering wireless sensor nodes to monitor structural health and to run household security systems [1]. To convert kinetic energy to electric energy, a typical method uses a linear resonator with a piezoelectric material. But a slight change of the excitation frequency will cause a dramatic reduction of response. Therefore, a nonlinear system was explored to broaden the frequency bandwidth to increase the efficiency of the energy harvesting. Differing from a linear resonator that has only one peak with narrow bandwidth in the frequency response, the nonlinear resonator can have a broad frequency bandwidth with a large response because of its hardening or softening behavior [2]. 
Among several approaches taken to apply nonlinearity, a bi-stable system using magnetic force is a common way $[3,4,5]$. The magnetic force creates cubic stiffness that makes the resonator act as a Duffing type oscillator. The performance of the bi-stable vibration energy harvester using piezoelectric material was studied under random excitation [6, 7]. The bistable system shows the advantage by increasing the amplitude at widespread low frequencies $[8,9]$. Other bi-stable vibration energy harvesters applying different transduction method such as electromagnetic $[10,11,12,13]$ were studied as well.

Vibration energy harvesters usually use a single degree-of-freedom system. Recently, several researchers proposed a multi-degree-of-freedom system to increase the output and broaden the frequency bandwidth $[14,15,16]$. The resonators with multiple degrees of freedom are designed to have close resonant frequencies combined together to widen the frequency bandwidth. Furthermore, by placing multiple resonators in different directions, a multi-degree-of-freedom system can be a directional energy harvester [17]. In multi-degree-offreedom systems, internal resonance has been reported. Xiong [18] and Chen [19] developed the two-degree-of-freedom system using a L-shape beam to achieve the internal resonance phenomeon. The authors' previous work [20, 21] investigated movable magnets in the conventional bi-stable system to increase the frequency bandwidth by activating the internal resonance. The transfer of energy from one mode of vibration into another because of the internal resonance phenomenon yields a large amplitude of oscillation at a wider frequency range.

Our previous study [21] of a similar double cantilever system focused on the internal resonance phenomenon. For the same system, in this study, we report the parametric resonance as an effective way to drive the system dynamics into a large distinctive response. Daqaq et al. [22] proposed a lumped-parameter nonlinear model that describes the first mode dynamics of a parametrically excited harvester. Their model considers the cantilever's geometric and inertia nonlinearity, but the main limitation of this is the requirement for a high excitation amplitude. Jia designed a vibration energy harvester by introducing a lever pivot and a pendulum mass to reduce the initial threshold value needed to trigger the parametric excitation [23]. This two-degree-of-freedom system is less constrained by the initial threshold value because the source of damping and primary oscillation acts in different freedoms. To apply resonant amplification of the base excitation, Jia proposed an auto parametrically excited vibration energy harvester [24]. Using a clamped-clamped beam whose natural frequency is twice that of the primary cantilever, the parametric resonator has a lower threshold value needed to trigger the parametric excitation, outperforming the same device driven into direct resonance by more than an order of magnitude. Besides two-degrees-of-freedom parametric vibration energy harvesters, the researcher added nonlinearity to the system by using magnets to increase the frequency bandwidth. Karami et al. [25] made a bi-stable system by incorporating a repelling magnetic force, which achieved a nonlinear phenomenon and generates sufficient power through a relatively large range of frequency.

In this work, we introduced magnetic nonlinearity to a two-degree-of-freedom system to address two main drawbacks of parametric resonators, which are high threshold excitation and narrow bandwidth. The system uses two piezoelectric cantilever beams, each with a magnet tip. One cantilever beam is in the horizontal plane and the other is in the vertical direction. The two piezoelectric cantilever beams are coupled by the magnetic force. The magnetic force reduces the threshold excitation level needed for the parametric resonance. 
Furthermore, the frequency bandwidth widens by the combination of direct and parametric resonances that show softening and hardening behavior. Thus, the introduced magnetic nonlinearity decreases the threshold excitation value needed for parametric resonance and widens the frequency bandwidth in which the harvester can operate efficiently.

The paper is organized this way. Section 3 gives the mathematical model of the nonlinear vibration energy harvesting. In Section 4, simulation and experiments results are obtained and compared. The effects of different parameters on the system response are studied in Section 5 , followed by a conclusion in Section 6 .

\section{Mathematical model}

\subsection{Governing equations}

The prototype of the parametric vibration energy harvester is shown in Figure 1. The energy harvester consists of a vertical cantilever beam with a magnetic tip facing another magnet attached to a horizontal cantilever beam, creating a coupled oscillator system. Both beams have piezoelectric layers into convert mechanical vibrations to electrical energy. The same poles of the magnets face each other, creating the repulsive magnetic force that couples the beams motions. The base excitation is along the longitudinal direction of the vertical beam. Under the base excitation, the vertical cantilever beam vibrates in the perpendicular direction of the base excitation. Therefore, the horizontal cantilever beam could show direct resonance and the vertical beam could undergo parametric resonance. In general, parametric resonators require a large threshold excitation to trigger the parametric resonance. To overcome this drawback, we introduce the repulsive magnetic force (compressive axial force) to decrease the natural frequency of the vertical beam to lower the initial threshold excitation level. In addition, by carefully designing the resonant frequency of the horizontal beam to be twice that of the vertical beam, while considering the effect of the magnetic force, the threshold excitation level for the parametric resonance decreases more. When the system is excited around the natural frequency of the horizontal beam, the vertical beam shows primary parametric resonance. This parametric excitation is caused both by the base motion as well as the magnetic force that couples the motion of the two beam. The magnetic force also acts on the vertical beam as a direct excitation source. Hence, the direct excitation because of the magnetic force and the parametric resonance of the vertical beam merge in the same frequency range and widen the frequency bandwidth with a simultaneous increase in the harvester amplitude.

The mathematical model of the parametric energy harvesting is obtained by a lumped model as shown in Figure 2. $X$ and $Y$ denote the deflection of the horizontal and vertical beams, respectively. $m_{1}$ and $m_{2}$ are the effective mass of the horizontal beam and vertical beam and $k_{1}$ and $k_{2}$ are the effective stiffness of the cantilever beam without the effect of the magnetic force, respectively. $c_{1}$ and $c_{2}$ are the equivalent damping for both beams. $d$ is the initial distance between two magnets, center to center. $x_{b}$ is the base excitation that is in the $X$ direction. Using Hamilton's principle as described in our previous work [21], the governing equations are obtained. However, the equations are different from our previous study as they include the parametric excitation term, which was neglected before. In addition, the setup is rotated vertically, and the effect of the weight of the horizontal beam is considered in the equation of motion. 


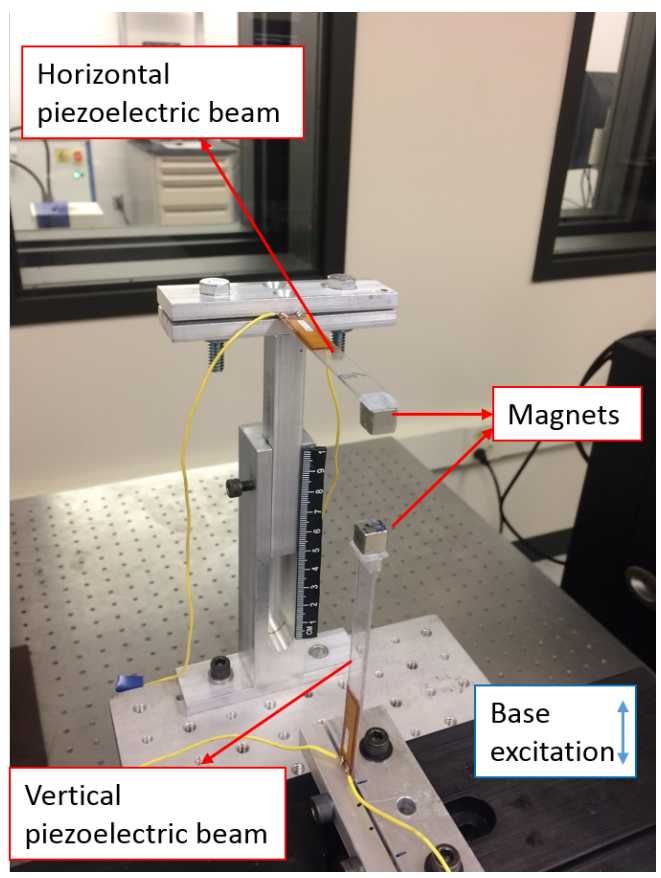

Figure 1: The parametric vibration energy harvester

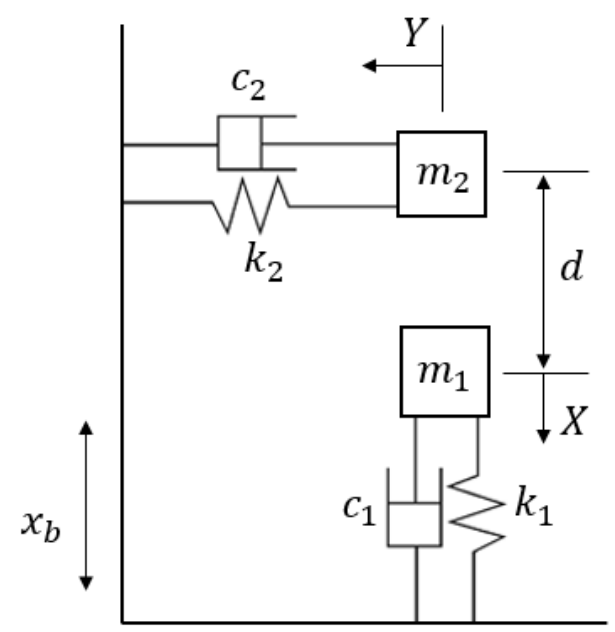

Figure 2: The lumped model of the parametric vibration energy harvesting.

$$
\begin{gathered}
m_{1} \ddot{X}+k_{1} X+c_{1} \dot{X}-F_{\text {magx }}-\theta_{e} v_{1}=-m_{1} \ddot{x}_{b}-m_{1} g \\
m_{2} \ddot{Y}+k_{2} Y+c_{2} \dot{Y}-F_{\text {magy }}-\theta_{e} v_{2}+m_{2} \ddot{x_{b}} Y=0 \\
c_{p} \dot{v}_{1}+\theta_{e} \dot{X}+\frac{v_{1}}{R}=0 \\
c_{p} \dot{v}_{2}+\theta_{e} \dot{Y}+\frac{v_{2}}{R}=0
\end{gathered}
$$

where $F_{\text {magx }}$ and $F_{\text {magy }}$ are the magnetic force in $X$ and $Y$ direction respectively, $c_{p}$ is the capacitance, $\theta_{e}$ is the coupling coefficient, and $R$ is the load resistance. The same piezoelectric 
material is used for both horizontal and vertical beams. Therefore, the $c_{p}, \theta_{e}$ and $R$ are the same for both beams. $v_{1}$ is the output voltage for the horizontal beam, and $v_{2}$ is the output voltage for the vertical beam. For the horizontal beam, the gravity of the effective mass $m_{1} g$ needs to be considered. For the vertical beam, the effect of gravity is ignored as it is negligible compared to the magnetic force and it is perpendicular to its vibration. The magnetic force is given from our previous work [21] as

$$
\begin{gathered}
F_{\text {magx }}=\frac{M_{c}}{D^{4}}\left(1-\frac{5 Y^{2}}{2 D^{2}}\right) \\
F_{\text {magy }}=\frac{M_{c} Y}{D^{5}}\left(1-\frac{5 Y^{2}}{2 D^{2}}\right) \\
M_{c}=\frac{3 \mu_{0} M^{2}}{2 \pi} \\
D=d+X
\end{gathered}
$$

where $M$ denotes the magnetization moment of a magnet, $\mu_{0}$ is the permeability of space. $D$ is the total distance between two magnets considering the initial distance $d$ and the deflection of the horizontal beam $X$. To get the dynamic response of the system and the resonant frequencies for both beams, the static equilibrium positions are obtained first. We assume the vertical beam is not buckled so the static position of the vertical beam is $y_{e}=0$. The static equilibrium position $x_{e}$ can be found as

$$
k_{1} x_{e}-\frac{M_{c}}{\left(d+x_{e}\right)^{4}}=-m_{1} g
$$

A Taylor expansion is used to expand $1 / D^{n}$ around the equilibrium positions. We write the total displacement as a summation of static deflection and dynamic amplitude $\left(X=x_{e}+x\right.$, $\left.Y=y_{e}+y\right)$. After the static terms are eliminated and fourth and higher orders terms are dropped, the dynamic governing equations are obtained as

$$
\begin{gathered}
\ddot{x}+\omega_{1}^{2} x+2 \mu_{1} \dot{x}+a_{1} y^{2}+a_{2} x y^{2}-\theta_{1} v_{1}=-\ddot{x}_{b} \\
\ddot{y}+\omega_{2}^{2} y+\ddot{x_{b}} y+2 \mu_{2} \dot{y}+a_{3} x y+a_{4} y^{3}-\theta_{2} v_{2}=0 \\
c_{p} \dot{v}_{1}+\theta_{e} \dot{x}+\frac{v_{1}}{R}=0 \\
c_{p} \dot{v}_{2}+\theta_{e} \dot{y}+\frac{v_{2}}{R}=0
\end{gathered}
$$

where $x$ and $y$ are the dynamic response of the horizontal and vertical cantilever beams, respectively. The coefficients are $\mu_{1}=\frac{c_{1}}{2 m_{1}}, \mu_{2}=\frac{c_{2}}{2 m_{2}}, a_{1}=\frac{5 M_{c}}{2 D_{e}^{6} m_{1}}, a_{2}=-\frac{15 M_{c}}{D_{e}^{7} m_{1}}, a_{3}=\frac{5 M_{c}}{D_{e}^{6} m_{2}}$, $a_{4}=\frac{5 M_{c}}{2 D_{e}^{7} m_{2}}, \theta_{1}=\frac{\theta_{e}}{m_{1}}, \theta_{2}=\frac{\theta_{e}}{m_{2}}$ and

$$
\omega_{1}^{2}=\frac{k_{1}+\frac{4 M_{c}}{D_{e}^{5}}}{m_{1}}
$$




$$
\omega_{2}^{2}=\frac{k_{2}-\frac{M_{c}}{D_{e}^{5}}}{m_{2}}
$$

Based on the equations (14-15), the resonant frequencies of two cantilever beams are functions of the distance between two magnets if the dimensions of the cantilever beams are determined. Hence, by controlling the initial distance $d$, the resonant frequency $\omega_{1}$ of the horizontal beam can be set to around twice the resonant frequency $\omega_{2}$ of the vertical beam. The equations (10-13) are solved numerically under the harmonic excitation. The simulated displacement and voltage frequency responses are presented in the Section 4.

\subsection{Analytical study of the coupled oscillator system}

In this section, the perturbation method of multiple scales is used to study the qualitative behavior of the coupled oscillator system analytically. This study expands previous work on coupled oscillator system [26]. Two time scales $T_{0}$ and $T_{1}$ are used [27]:

$$
\begin{gathered}
T_{0}=t \\
T_{1}=\varepsilon t
\end{gathered}
$$

where $\varepsilon$ is a scaling parameter. Let $-\ddot{x}_{b}=\varepsilon f \cos (\Omega t)$, the governing equations (10-11) become:

$$
\begin{gathered}
\left.\ddot{x}+\omega_{1}^{2} x+\varepsilon\left(2 \mu_{1} \dot{x}+a_{1} y^{2}+a_{2} x y^{2}-\theta_{1} v_{1}(t)\right)\right)=\varepsilon f \cos (\Omega t) \\
\ddot{y}+\omega_{2}^{2} y+\varepsilon\left(2 \mu_{2} \dot{y}+a_{3} x y+a_{4} y^{3}-f \cos (\Omega t) y-\theta_{2} v_{2}(t)\right)=0
\end{gathered}
$$

The next step is to assume an asymptotic series solution for $x(t), y(t)$ and $v(t)$. In this case, a two-term expansion is assumed:

$$
\begin{gathered}
x(t)=x_{1}\left(T_{0}, T_{1}\right)+\varepsilon x_{2}\left(T_{0}, T_{1}\right) \\
y(t)=y_{1}\left(T_{0}, T_{1}\right)+\varepsilon y_{2}\left(T_{0}, T_{1}\right) \\
v_{1}(t)=v_{11}\left(T_{0}, T_{1}\right)+\varepsilon v_{12}\left(T_{0}, T_{1}\right) \\
v_{2}(t)=v_{21}\left(T_{0}, T_{1}\right)+\varepsilon v_{22}\left(T_{0}, T_{1}\right)
\end{gathered}
$$

We use chain rules and drop orders higher than one to get time derivatives, then we plug time derivatives into the governing equations and gather coefficients of orders up to one yields

$\varepsilon^{0}$ order:

$$
\begin{aligned}
& \frac{\partial^{2} x_{1}}{\partial T_{0}^{2}}+\omega_{1}^{2} x_{1}=0 \\
& \frac{\partial^{2} y_{1}}{\partial T_{0}^{2}}+\omega_{2}^{2} y_{1}=0
\end{aligned}
$$

$\varepsilon^{1}$ order:

$$
\left.\frac{\partial^{2} x_{2}}{\partial T_{0}^{2}}+\omega_{1}^{2} x_{2}=-2 \mu_{1} \frac{\partial x_{1}}{\partial T_{0}}-2 \frac{\partial^{2} x_{1}}{\partial T_{0} \partial T_{1}}-a_{1} y_{1}^{2}-a_{2} x_{1} y_{1}^{2}+f \cos \left(\Omega T_{0}\right)\right)+\theta_{1} v_{11}
$$




$$
\frac{\partial^{2} y_{2}}{\partial T_{0}^{2}}+\omega_{2}^{2} y_{2}=-2 \mu_{2} \frac{\partial y_{1}}{\partial T_{0}}-2 \frac{\partial^{2} y_{1}}{\partial T_{0} \partial T_{1}}-a_{3} x_{1} y_{1}-a_{4} y_{1}^{3}+f \cos \left(\Omega T_{0}\right)+\theta_{2} v_{21}
$$

The solutions of (18-19) are given by:

$$
\begin{aligned}
& x_{1}=P_{1}\left(T_{1}\right) e^{i \omega_{1} T_{0}}+\bar{P}_{1}\left(T_{1}\right) e^{-i \omega_{1} T_{0}} \\
& y_{1}=P_{2}\left(T_{1}\right) e^{i \omega_{2} T_{0}}+\bar{P}_{2}\left(T_{1}\right) e^{-i \omega_{2} T_{0}}
\end{aligned}
$$

To study the coupled motion of our two-degree of freedom system we use the internal resonance relationship to eliminate the secular terms in the equations. Considering the case:

$$
\begin{aligned}
& \Omega=2 \omega_{2}+\varepsilon \sigma_{1} \\
& \omega_{1}=2 \omega_{2}+\varepsilon \sigma_{2}
\end{aligned}
$$

The equations (22-23) give the reason analytically that we chose frequency ratio 1 to 2 to trigger the parametric resonance of the vertical beam. When we have an internal resonance condition, more nonlinear terms are given as below:

$$
\begin{gathered}
2 \mu_{1} i \omega_{1} P_{1}+2 i \omega_{1} \frac{\partial P_{1}}{\partial T_{1}}+a_{1} P_{2}^{2} e^{-i \sigma_{2} T_{1}}+2 a_{2} P_{1} P_{2} \bar{P}_{2}-\frac{f}{2} e^{i\left(\sigma_{1}-\sigma_{2}\right) T_{1}}+i \frac{\omega_{2} P_{2} \theta_{1} \theta_{e}}{\frac{1}{R}+i \omega_{2} c_{p}}=0 \\
2 \mu_{2} i \omega_{2} P_{2}+2 i \omega_{2} \frac{\partial P_{2}}{\partial T_{1}}+a_{3} P_{1} \bar{P}_{2} e^{i \sigma_{2} T_{1}}+3 a_{4} P_{2}^{2} \bar{P}_{2}-\frac{1}{2} f e^{i \sigma_{1} T_{1}}+i \frac{\omega_{2} P_{2} \theta_{2} \theta_{e}}{\frac{1}{R}+i \omega_{2} c_{p}}=0
\end{gathered}
$$

The complex $P_{1}$ and $P_{2}$ are described in polar form as

$$
\begin{aligned}
& P_{1}\left(T_{1}\right)=\frac{1}{2} p_{1}\left(T_{1}\right) e^{i \varphi_{1}\left(T_{1}\right)} \\
& P_{2}\left(T_{1}\right)=\frac{1}{2} p_{2}\left(T_{1}\right) e^{i \varphi_{2}\left(T_{1}\right)}
\end{aligned}
$$

The polar forms are substituted into equations (24) and (25). Set the real part and the imaginary part to zero respectively and for the steady-state response, setting $\frac{\partial p_{1}}{\partial T_{1}}=\frac{\partial p_{2}}{\partial T_{1}}=$ $\frac{\partial \gamma_{1}}{\partial T_{1}}=\frac{\partial \gamma_{2}}{\partial T_{1}}=0$ yields

$$
\begin{gathered}
4 \omega_{1} \mu_{1} p_{1}+a_{1} p_{2}^{2} \sin \left(\gamma_{1}\right)-2 f \sin \left(\gamma_{2}\right)+4 \omega_{1} E_{11} p_{1}=0 \\
a_{1} p_{2}^{2} \cos \left(\gamma_{1}\right)-2 f \cos \left(\gamma_{2}\right)-4 \omega_{1} p_{1}\left(\sigma_{1}-\sigma_{2}\right)+a_{2} p_{1} p_{2}^{2}+4 \omega_{1} E_{12} p_{1}=0 \\
-4 \omega_{2} \mu_{2}+f \sin \left(\gamma_{2}-\gamma_{1}\right)+a_{3} p_{1} \sin \left(\gamma_{1}\right)+4 \omega_{2} E_{21}=0 \\
2 a_{3} p_{1} \cos \left(\gamma_{1}\right)+3 a_{4} p_{2}^{2}-2 f \cos \left(\gamma_{2}-\gamma_{1}\right)-4 \omega_{2} \sigma_{1}+8 \omega_{2} E_{22}=0
\end{gathered}
$$

where

$$
\begin{gathered}
\gamma_{1}=2 \varphi_{2}-\varphi_{1}-\sigma_{2} T_{1} \\
\gamma_{2}=\sigma_{1} T_{1}-\sigma_{2} T_{1}-\varphi_{2}
\end{gathered}
$$




$$
\begin{aligned}
& E_{11}=-\frac{\frac{\theta_{1} \theta_{e}}{2 R}}{\frac{1}{R^{2}}+\omega_{1}^{2} c_{p}^{2}} \\
& E_{12}=\frac{\frac{\theta_{1} \theta_{e} \omega_{1} c_{p}}{2}}{\frac{1}{R^{2}}+\omega_{1}^{2} c_{p}^{2}} \\
& E_{21}=-\frac{\frac{\theta_{2} \theta_{e}}{2 R}}{\frac{1}{R^{2}}+\omega_{2}^{2} c_{p}^{2}} \\
& E_{22}=\frac{\frac{\theta_{2} \theta_{e} \omega_{2} c_{p}}{2}}{\frac{1}{R^{2}}+\omega_{2}^{2} c_{p}^{2}}
\end{aligned}
$$

The output voltages are

$$
\begin{aligned}
& v_{1}=\frac{\omega_{1} \theta_{e}}{\sqrt{1 / R^{2}+\omega_{1}^{2} c_{p}^{2}}} p_{1} \\
& v_{2}=\frac{\omega_{2} \theta_{e}}{\sqrt{1 / R^{2}+\omega_{2}^{2} c_{p}^{2}}} p_{2}
\end{aligned}
$$

By solving equations (26-31), the frequency responses can be obtained. The results are presented in Section 4.

\subsection{Analytical study of a conventional bi-stable system}

One of the main points of our novel design is that our two-degree-of-freedom coupled oscillator system significantly decreases the initial threshold excitation level required to trigger the parametric resonance. To understand how our coupled oscillator system reduces the initial threshold excitation needed to induce parametric resonance, we discuss a conventional bi-stable harvester with magnetic nonlinearity. A schematic of the conventional harvester is shown in Figure 3.

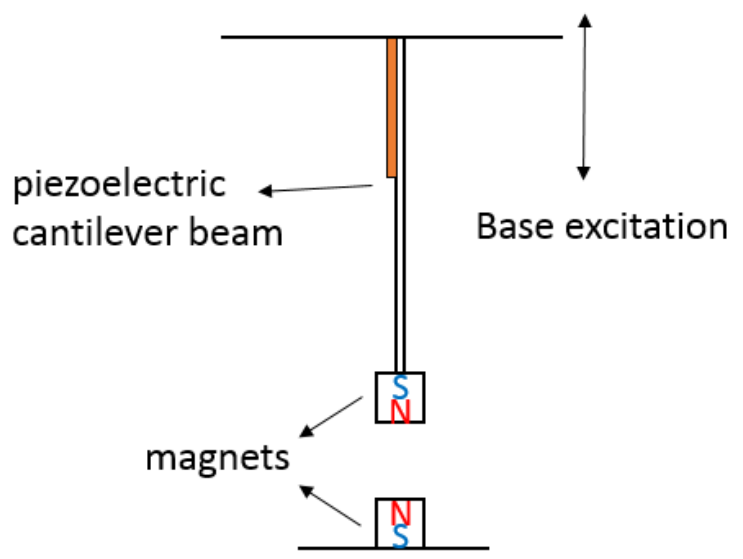

Figure 3: A convential bi-stable system under parametric excitation. 
Assume this bi-stable system is excited by a parametric excitation whose direction is parallel with the longitudinal direction of the beam. The governing equation [9] is

$$
\ddot{y}_{c}+2 \mu_{c} \dot{y}_{c}+\omega_{n c}^{2} y_{c}-A_{a} \cos (\Omega t) y_{c}+\alpha y_{c}^{3}=0
$$

where

$$
\begin{gathered}
\omega_{n c}=\frac{k_{1 c}}{m} \\
k_{1 c}=k-\frac{M_{c}}{D_{c}^{5}} \\
\alpha=\frac{k_{3 c}}{m} \\
k_{3 c}=\frac{5 M c}{2 D_{c}^{7}}
\end{gathered}
$$

and $y_{c}$ is the deflection of the beam, $\mu_{c}$ is the equivalent damping ratio including the effect of the piezoelectric strip, $\Omega$ denotes the excitation frequency and $A_{a}$ is the acceleration amplitude. $\omega_{n c}$ is the natural frequency of the beam, and $\alpha$ is the cubic nonlinearity. $k$ is the effective stiffness of the beam considering the effect of the magnetic tip, $k_{1 c}$ is the linear

stiffness of the system that changes with the distance $D_{c}$ between magnets, $M_{c}=\frac{3 \mu_{0} M^{2}}{2 \pi}$, where $M$ is the magnetization moment and $\mu_{0}$ is the permeability of space. To solve the equation (32) analytically, the perturbation method mentioned in section 3.2 is followed. For the steady-state response, we obtain the analytical solution for the amplitude as excitation frequency changes around twice the natural frequency:

$$
p_{c}^{2}=\frac{4 \sigma \omega_{n c}}{3 \alpha} \pm \frac{2}{3 \alpha} \sqrt{A_{a}^{2}-16 \omega_{n c}^{2} \mu_{c}^{2}}
$$

In this conventional bi-stable system, the excitation level to trigger the parametric resonance must be $A_{a}>4 \omega_{n c} \mu_{c}$, where the natural frequency $\omega_{n c}$ depends on the initial distance between two magnets. Thus, this relationship indicates that to have a lower threshold, the natural frequency and the damping ratio should be low. Comparison between the conventional bi-stable system and our coupled oscillator system design is studied in the following section.

\section{Results and discussions}

\subsection{Model verification}

The experimental setup is shown in Figure 4. The prototype of the energy harvester is placed on the shaker (VTB 100) to apply the harmonic base excitation. Data is acquired with NI-6251 (DAQ) from National Instruments connected to Matlab software. The DAQ sends the input signal through an amplifier to the shaker at the specific excitation level and sweeps a range of frequency at $0.1 \mathrm{~Hz} / \mathrm{s}$. Simultaneously, the data from the accelerometer and piezoelectric strip are sent to the Matlab to obtain a time series and frequency response curve after signal processing. The accelerometer (PCB 352A24) measured the base excitation of the shaker. The horizontal cantilever beam and vertical cantilever beam are made of a 


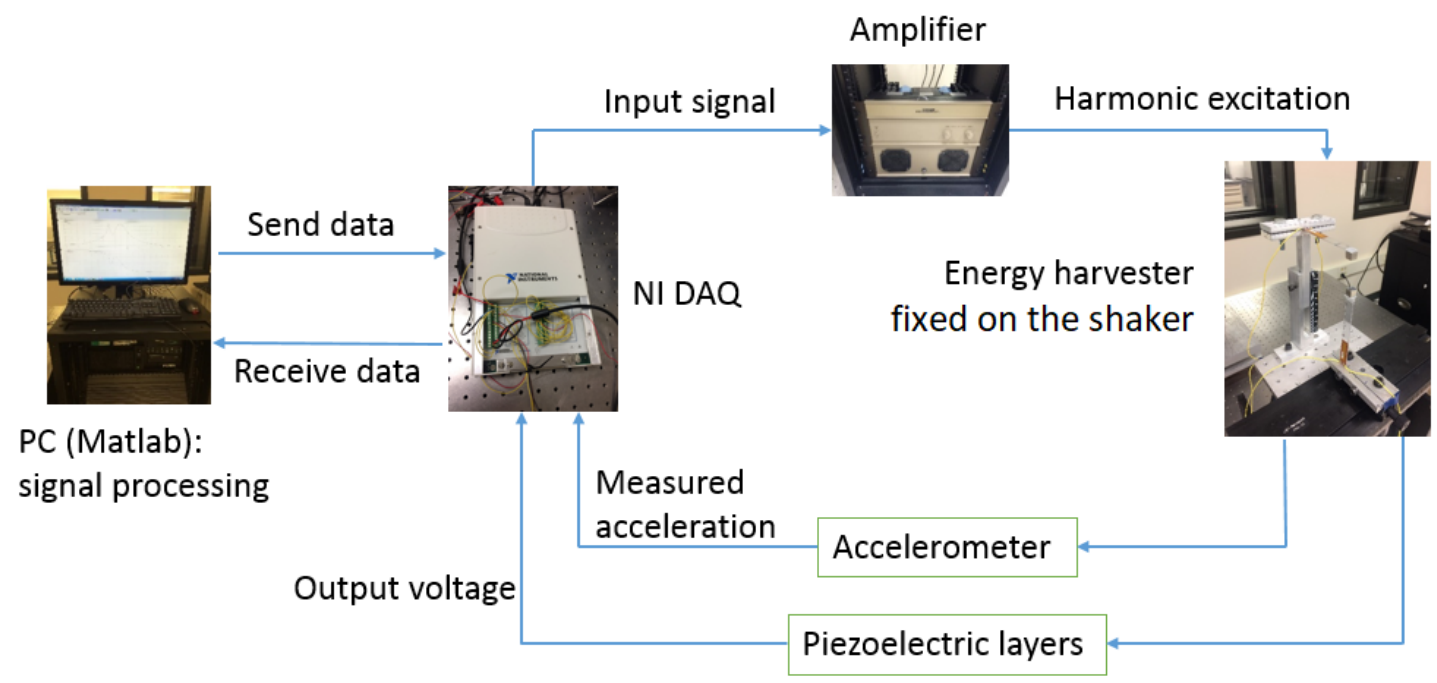

Figure 4: Schematic of the experimental setup for the energy harvesting.

polymeric (Makrolon polycarbonate) material with a modulus of elasticity of $2.34 \mathrm{GPa}$, and partially covered by the piezoelectric material (Smart Materials Corp., model: M2807P2). Two cubic permanent magnets with sides of $8 \mathrm{~mm}$ with magnetization moment of $0.53 \mathrm{Am}^{2}$ are attached to the tip of each beam.

The parameters used in the simulation and experiment are shown in Table 1 . The default base excitation level is set to be $0.4 \mathrm{~g}$. Without the effect of magnetic force, the natural frequency of the horizontal beam is $10.03 \mathrm{~Hz}$ and the natural frequency of the vertical beam is $7.02 \mathrm{~Hz}$. When the distance between the two magnets is large, the deflections of the two beams are not coupled by the magnetic force. The horizontal beam generates the electrical energy because of the piezoelectric element in the same way as a linear resonator. The vertical beam does not oscillate, because the excitation level is not large enough to trigger the parametric resonance. The presence of the piezoelectric strip on the vertical beam makes it even worse as it adds damping to the system demanding a high threshold level for parametric resonance. As we decrease the distance between the two magnets, the coupling between the two beam motions becomes stronger. Based on equations (14-15), the natural frequency of the horizontal beam, $\omega_{1}$ increases. On the contrary, the natural frequency of the vertical beam, $\omega_{2}$ decreases. At a distance when the resonant frequency $\omega_{1}$ is close to $2 \omega_{2}$, the parametric resonance of the vertical beam is triggered.

Numerically simulated frequency responses of the horizontal and vertical beams when $d=34 \mathrm{~mm}$ at $0.4 \mathrm{~g}$ are shown in Figure $5 \mathrm{a}$ and $5 \mathrm{~b}$, respectively. The corresponding output voltage of two beams are given in Figure $6 \mathrm{a}$ and $6 \mathrm{~b}$. These results were obtained by numerically solving equations (1-4). At $d=34 \mathrm{~mm}, \omega_{1}=11.61 \mathrm{~Hz}$ and $\omega_{2}=5.97 \mathrm{~Hz}$. Both forward and backward frequency sweeps were conducted to reveal the hysteresis phenomenon. As expected, the parametric resonance of the vertical beam creates a larger amplitude than that of the direct resonance of the horizontal beam. Similarly, the output voltage generated by the vertical beam is larger than that of the horizontal beam. The frequency response of the horizontal beam (Figure 5a and Figure 6a) shows it has a softening behavior. This agrees 


\begin{tabular}{lr}
\hline Parameters & Values \\
\hline \hline Effective mass of horizontal beam and magnet, $m_{1}$ & $8.6 \mathrm{~g}$ \\
Effective mass of vertical beam and magnet, $m_{2}$ & $5.5 \mathrm{~g}$ \\
Stiffness of horizontal beam, $k_{1}$ & $34.05 \mathrm{~N} / \mathrm{m}$ \\
Stiffness of vertical beam, $k_{2}$ & $7.72 \mathrm{~N} / \mathrm{m}$ \\
Damping of horizontal beam, $c_{1}$ & $0.029 \mathrm{Ns} / \mathrm{m}$ \\
Damping of vertical beam, $c_{2}$ & $0.018 \mathrm{Ns} / \mathrm{m}$ \\
Coupling coefficient, $\theta_{e}$ & $-5.4 \times 10^{-6} \mathrm{~N} / \mathrm{V}$ \\
Load resistance, $R$ & $1.8 \times 10^{6} \Omega$ \\
Capacitance, $c_{p}$ & $10 \times 10^{-9} \mathrm{~F}$ \\
magnetization moment, $M$ & $0.53 \mathrm{Am}$ \\
\hline
\end{tabular}

Table 1: Energy harvester parameters used in simulation and experiment.

with equation (10) where the cubic term is negative. The frequency response of the vertical beam (Figure 5b and Figure 6b) is more complicated as it combines two nonlinear phenomena. Hysteresis is observed, caused by the softening behavior induced by the horizontal beam. However, the slope of the softening response is reduced because it is combined with the hardening parametric resonance of the vertical beam, which has advantages for energy harvesting as the amplitude stays on a high branch. The cause of the hardening behavior of the vertical beam is the positive cubic term in equation (11). The combined nonlinearity results in wider frequency bandwidth and unique large vibration amplitudes compared to direct resonance. The absence of the multiple amplitudes for most of the frequency bandwidth range is a superior property of this design compared to conventional bi-stable resonators.

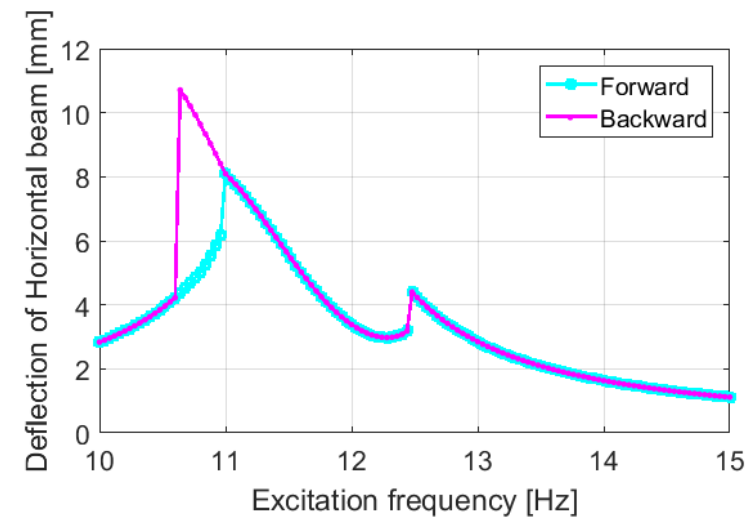

(a)

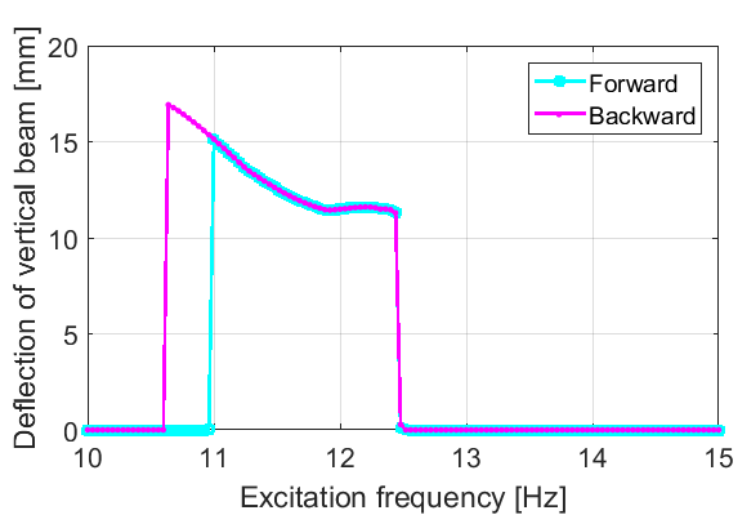

(b)

Figure 5: Frequency response of two beams at $0.4 \mathrm{~g}$ when $d=34 \mathrm{~mm}$ by simulation. a) the horizontal beam, b) the vertical beam.

In addition to numerically solving the equations using long time integration, we used the perturbation method of multiple scales as in Section 3.2 to solve the equations of motion. 


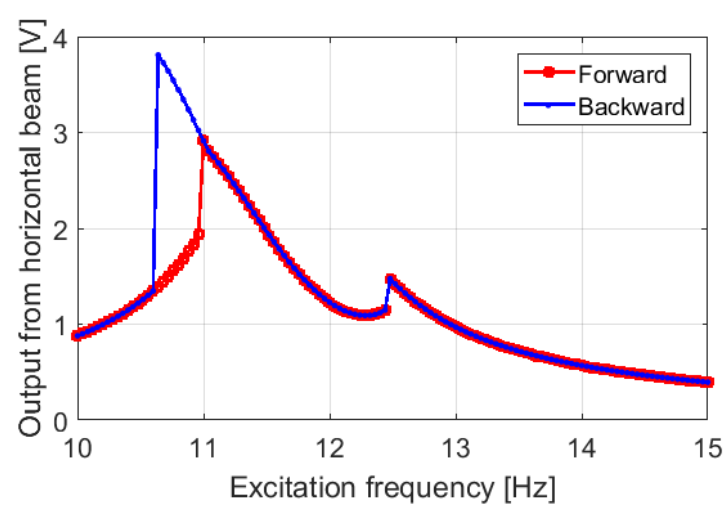

(a)

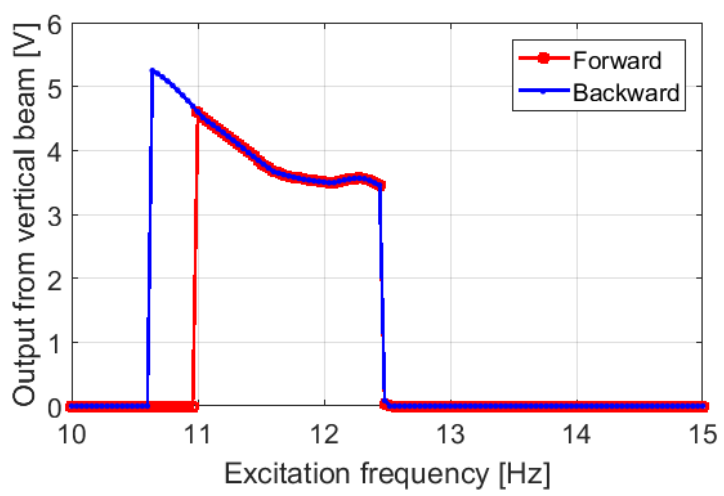

(b)

Figure 6: Output voltage of two beams at $0.4 \mathrm{~g}$ when $d=34 \mathrm{~mm}$ by simulation. a) the horizontal beam, b) the vertical beam.

Figure 7 shows the comparison of numerical and analytical solutions of the parametric resonance for the vertical beam. It is observed the analytical solution is in close agreement with the numerical solution. The small difference may come from the assumption of the asymptotic series solution, because some higher order terms are eliminated. Regardless of the small difference, the perturbation method of multiple scales gives a reliable solution of the governing equations.

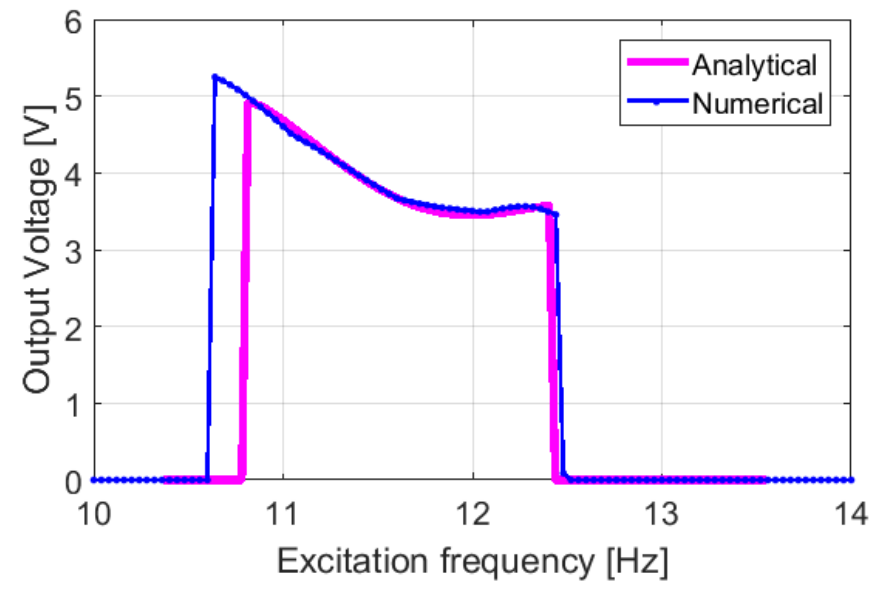

Figure 7: Output voltage when $d=34 \mathrm{~mm}$ at excitation level of $0.4 \mathrm{~g}$.

Figure $8 \mathrm{a}$ and $8 \mathrm{~b}$ show the experimental output voltage for the horizontal beam and vertical beam respectively with excitation level of $0.4 \mathrm{~g}$ when $d=34 \mathrm{~mm}$. The forward and backward sweeps represent the frequency output voltage. There is a good agreement between the experimental and simulation results. It is observed that there is hysteresis phenomenon because of the softening behavior. Also, the hardening parametric resonance combined with the softening behavior broadened the frequency bandwidth.

To examine the difference of the direct resonance of the horizontal beam and parametric resonance of the vertical beam, the simulated time responses at $12 \mathrm{~Hz}$ are depicted in Figure 


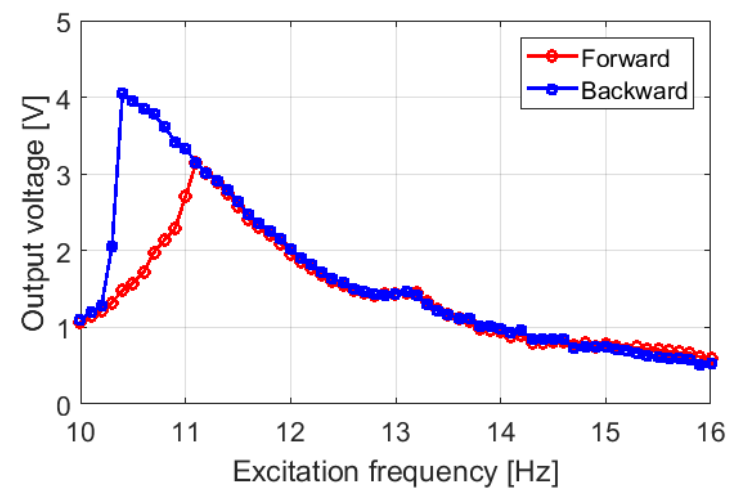

(a)

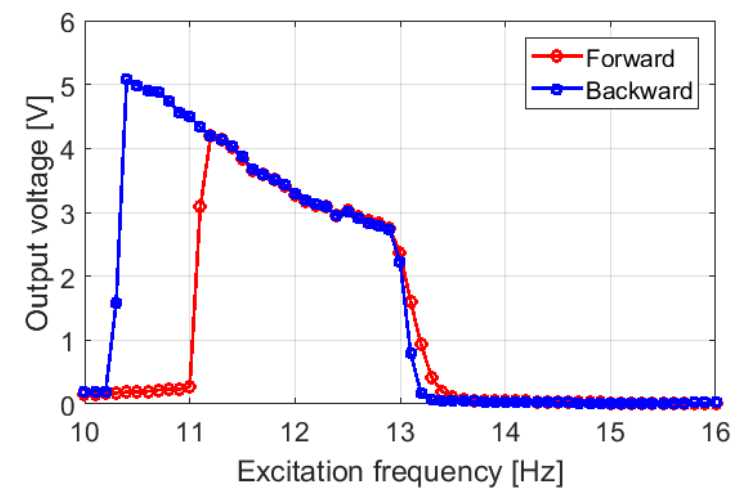

(b)

Figure 8: Output voltage of two beams at $0.4 \mathrm{~g}$ when $d=34 \mathrm{~mm}$ by experiment a) horizontal beam, b) vertical beam.

$9 \mathrm{a}$ and $9 \mathrm{~b}$. The period of the direct resonance of the horizontal beam is $0.0833 s$ and the resonant frequency is $12 \mathrm{~Hz}$ that is equal to the excitation frequency. For the vertical beam, the period of oscillation is $0.166 \mathrm{~s}$ and the frequency is $6 \mathrm{~Hz}$. That means the vertical beam motion is not from direct excitation caused by magnetic force, but rather it is parametric resonance. Because if it was direct excitation, the frequency of oscillation of the vertical beam would have been $12 \mathrm{~Hz}$, but here its frequency is $6 \mathrm{~Hz}$, which is the resonance frequency of the vertical beam. That means the beam responds at half the excitation frequency and is an indication of the activation of parametric resonance in the vertical beam. The parametric excitation of the vertical beam is caused by both the base motion and the magnetic force.

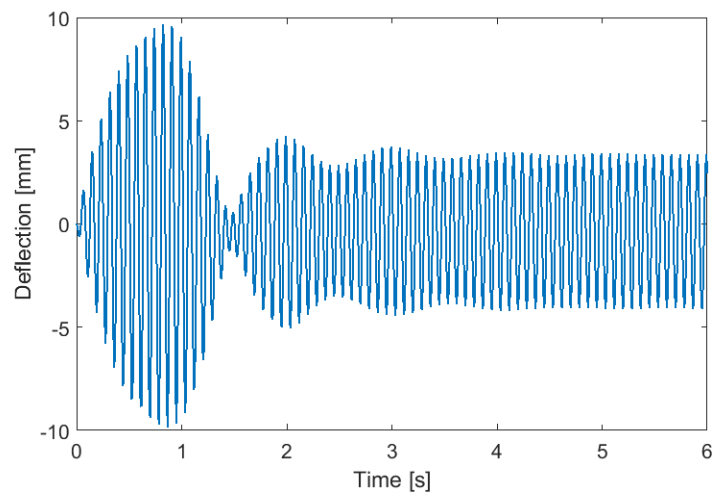

(a)

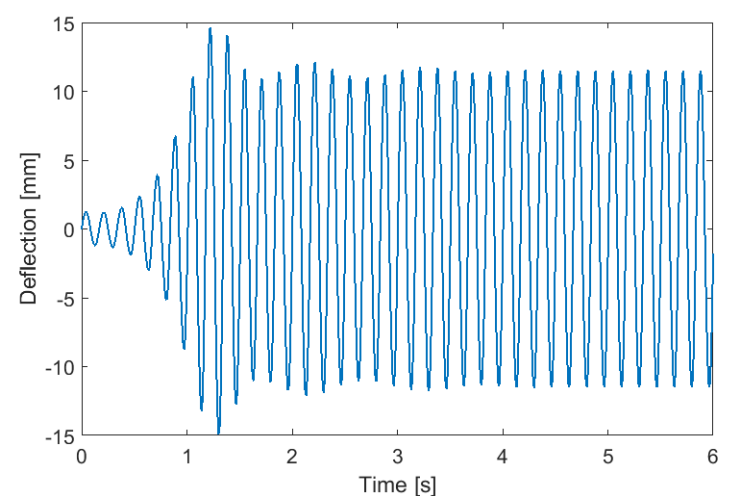

(b)

Figure 9: Time response of two beams at $12 \mathrm{~Hz}$ at $0.4 \mathrm{~g}$ when $d=34 \mathrm{~mm}$ a) horizontal beam, b) vertical beam.

\subsection{Comparison of the coupled oscillator system to the conventional bi-stable system}

To show the advantage of low threshold excitation in our design, we compared the performance of the conventional bi-stable system and proposed coupled oscillator system based 
on the analysis in the Sections 3.2 and 3.3. First, in the case of a conventional bi-stable system, the excitation level to trigger the parametric resonance must be $A_{a}>4 \omega_{n c} \mu_{c}$ according to the equation (33). We use the same parameters shown in Table 1 for the conventional bi-stable system. The parametric resonant frequency and initial threshold excitation level as the distance between magnets varies are shown in Figure 10a and 10b. When the distance is large, the system acts similar to a single cantilever beam without the effect of the magnetic force. It demands at least a $5.5 \mathrm{~g}$ excitation level to trigger the parametric resonance. When the distance decreases, the initial excitation level decreases as well. However, the minimum threshold value for the conventional bi-stable system is $1.6 \mathrm{~g}$, which is four times larger than our proposed coupled oscillator system that shows parametric resonance at $0.4 \mathrm{~g}$ as shown in Figure 8b.

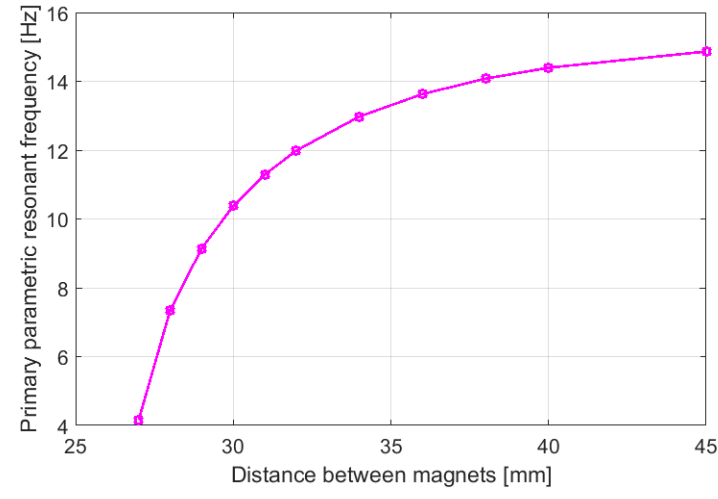

(a)

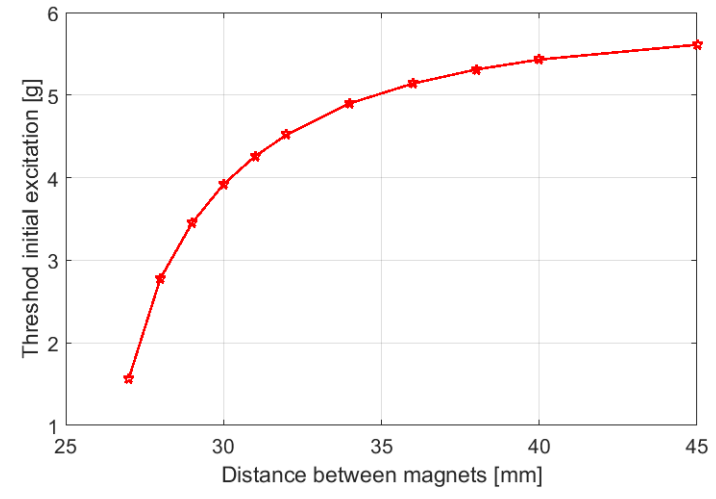

(b)

Figure 10: For the conventional bi-stable system including a cantilever beam with a magnetic tip and fixed magnet a) Primary parametric resonant frequency changing with the distance between two magnets, b) Initial threshold excitation level changing with the distance.

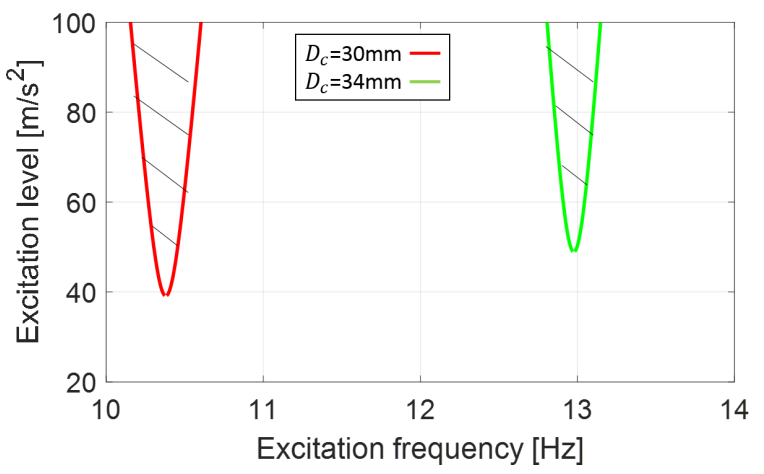

(a)

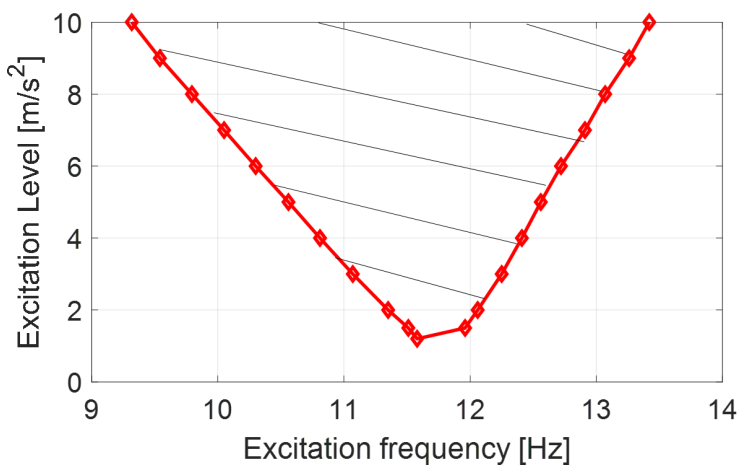

(b)

Figure 11: a) The two instability tongues of the conventional bi-stable system for $D_{c}=30 \mathrm{~mm}$ and $\left.D_{c}=34 \mathrm{~mm} ., \mathrm{b}\right)$ instability tongue for the coupled oscillator system when $d=34 \mathrm{~mm}$.

To show the superior performance of the coupled oscillator system, we used the analytical solutions in Section 3.2. The solutions provide insight into the regions where the parametric 
resonance can be excited. Using equations (33) and (26-29), the instability tongues for a conventional bi-stable system and coupled oscillator system obtained and depicted in Figures $11 \mathrm{a}$ and 11b. When the system works in the shaded zones, the parametric resonance is triggered. Therefore, the lowest value of the instability tongue curve is the initial parametric excitation. For the conventional bi-stable system, the base excitation level to initiate the parametric resonance varies with distance between magnets and the parametric resonance area (shaded area) is much narrower than the coupled oscillator system. The coupled system has both a low threshold excitation value and broader frequency range. That means the chances for large distinct parametric resonance of the system are much higher for the coupled system.

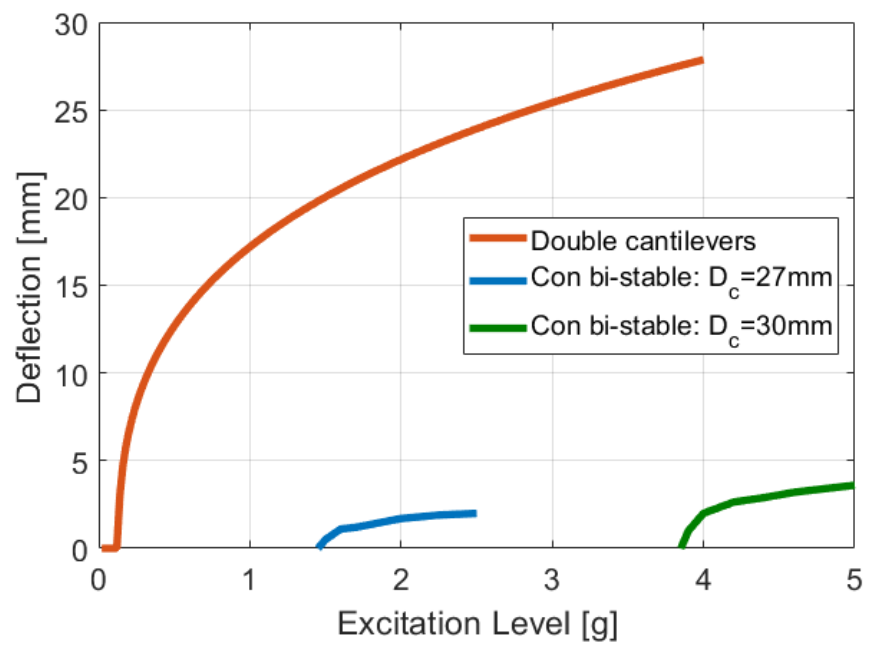

Figure 12: Steady-state parametric resonance amplitude as the excitation level at $12 \mathrm{~Hz}$ when $d=34 \mathrm{~mm}$ for coupled oscillator system and the conventional bi-stable system when distance between magnets are $D_{c}=27 \mathrm{~mm}$ and $D_{c}=30 \mathrm{~mm}$.

The other disadvantage for the conventional bi-stable system is that the parametric resonance amplitude is very small when the magnets are too close because of the strong nonlinearity of the system. From Figure 10b, we observe that the threshold excitation decreases when the distance between magnets reduces, but the deflection decreases as well. If the distance between the magnets increases, the amplitude can be larger but it requires harder force to trigger the parametric resonance. Our coupled oscillator system resolves this issue as the distance between magnets is variable and that reduces the stiffening effect from the magnets. The parametric resonance amplitudes as a function of excitation level are depicted for the coupled and conventional system in Figure 12, the threshold excitation to trigger the parametric resonance of the coupled oscillator system is low and the parametric deflection is much higher than the conventional bi-stable system. Thus, the proposed design takes advantage of the magnetic nonlinearity to lower the threshold without stiffening the system.

\section{Parametric study}

In this section, we study the performance of the proposed energy harvester by comparing the experimental and numerical results varying different parameters. When the excitation 
level is decreased to $0.3 \mathrm{~g}$, the parametric resonance is still obtained. Figure 13a and $13 \mathrm{~b}$ show the output voltage changes with the excitation level. In Figure 13b, it is observed that the frequency bandwidth increases as the excitation level increases. The slope of the softening behavior does not change with excitation level; however, as the excitation level increases, the output shows stronger hardening behavior because of the growth of the parametric resonance. This growth flattens the frequency bandwidth and increases the output at the same time.

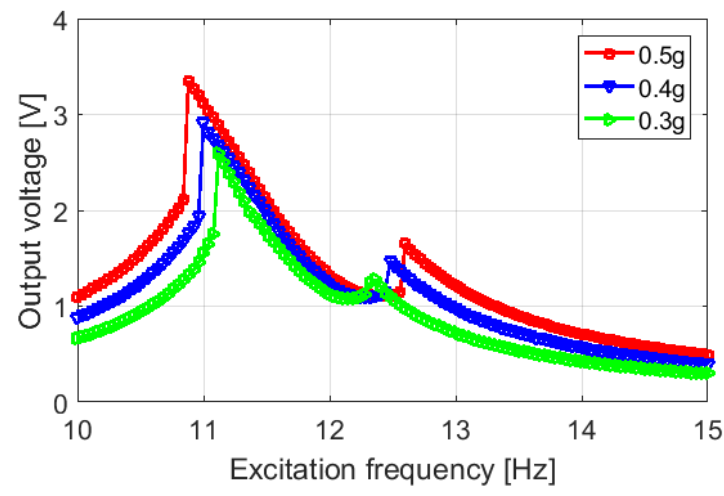

(a)

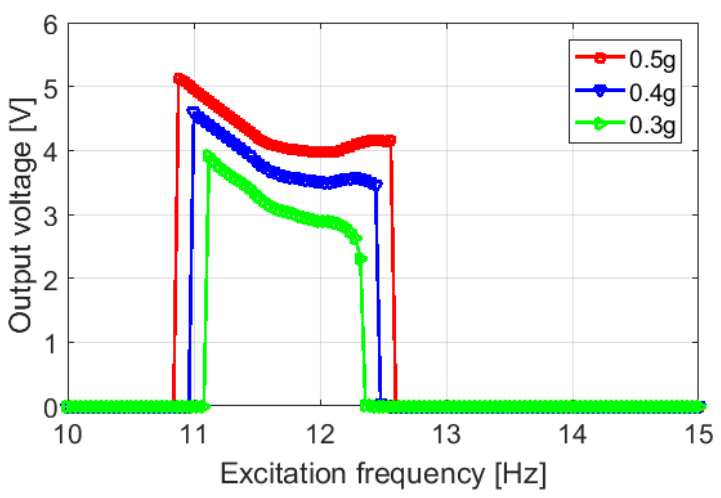

(b)

Figure 13: Simulated output voltage for different excitation level when disatnce between magnets is $d=$ $34 \mathrm{~mm}$ a) horizontal beam, b) vertical beam.

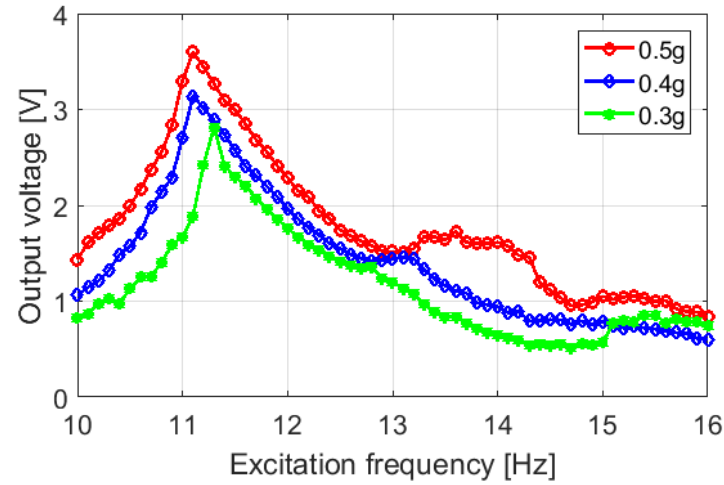

(a)

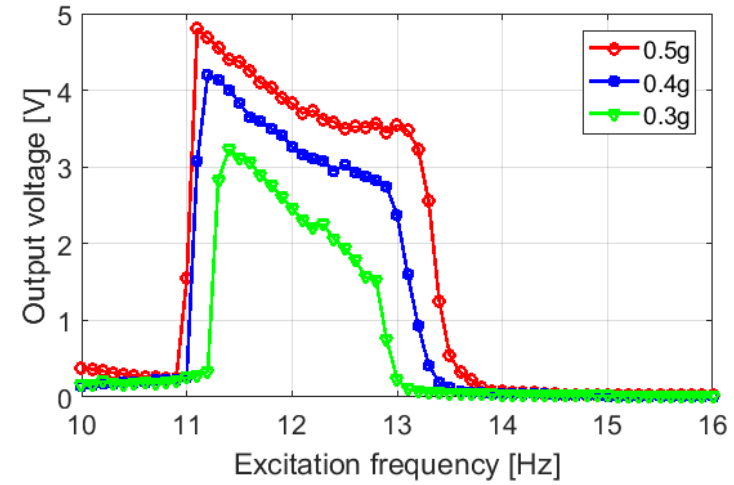

(b)

Figure 14: Measured output voltage for different excitation level when the distance between magnets is $d=34 \mathrm{~mm}$ a) horizontal beam, b) vertical beam.

To experimentally examine the effect of excitation level on the frequency response, three excitation levels of $0.3 \mathrm{~g}, 0.4 \mathrm{~g}$ and $0.5 \mathrm{~g}$ are used. We obtained the same behavior as predicted by the simulations. Higher excitation levels result in wider frequency bandwidth for the vertical beam (Figure 14b). The parametric resonance depends on the excitation level. If the excitation level is low, the parametric resonance is weak compared to direct resonance with the softening behavior. As predicted by the model, the horizontal beam shows softening behavior (Figure 14a). 
As the input level for energy harvesting is uncontrollable, to widen the bandwidth, the distance between the two magnets is decreased (from $d=34 \mathrm{~mm}$ to $d=32 \mathrm{~mm}$ ) as shown in Figures 15b and 15a. As the distance decreases to $d=32 \mathrm{~mm}$, the growth of parametric resonance and the hardening effect is stronger even for low excitation level $0.3 \mathrm{~g}$ as shown in Figure 15a. This is expected because as the magnets get closer to each other, the magnetic force causes a stronger coupling of the motions of the two beams. Thus, at the lower initial distance between the two magnets, the parametric resonance is triggered even at a lower excitation level and can even produce a larger output voltage. This result is opposite to conventional bi-stable system, which has a lower amplitude at the smaller distance between magnets. The theoretical results are in good agreement with experimental results, which means the nonlinear phenomenon is captured accurately by the model. However, the model underestimates the frequency bandwidth slightly.

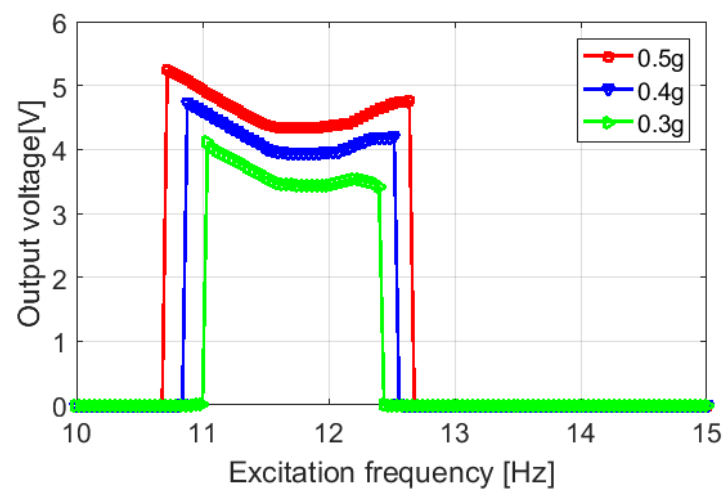

(a)

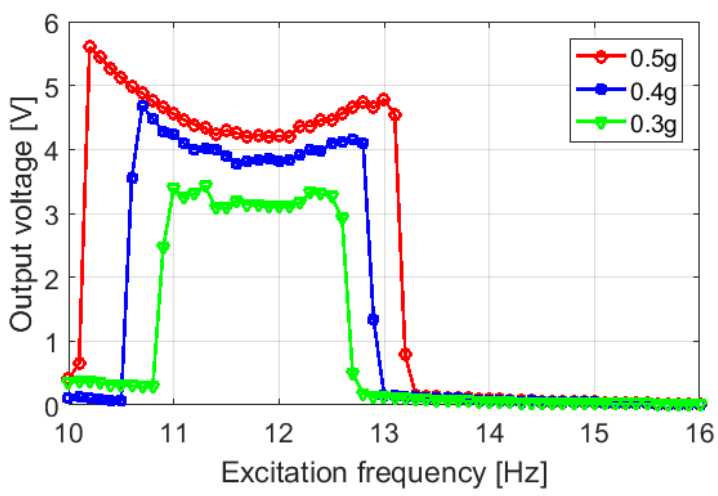

(b)

Figure 15: Output voltage results for different excitation level for vertical beam when distance between magnets is $d=32 \mathrm{~mm}$ a) Theoretical, b) Experimental.

The distance between magnets has a critical effect on the behavior of the system. Figure 16a and 16b illustrate how varying distances between the magnets affect output over a range of excitation frequencies. For the horizontal beam, in Figure 16a, the output decreases when the distance decreases. The repulsive magnetic force is large when the distance is small. This large magnetic force limits the motion of the horizontal beam. Hence the output voltage of the horizontal beam is smaller at shorter distances. However, the output voltage from the vertical beam is large when the distance $(d)$ is small because the parametric resonance dominates the response. In fact, the parametric resonance grows as the distance shrinks. Even though the maximum value of output voltage for the parametric resonance (vertical beam) is similar to that of the direct resonance (horizontal beam), the parametric resonances have a broader frequency bandwidth. If we decrease the distance to less than $30 \mathrm{~mm}$, the response will be chaotic and unpredictable. When $d=30 \mathrm{~mm}$, the resonant frequencies of the horizontal and vertical beams are $\omega_{1}=12.08 \mathrm{~Hz}$ and $\omega_{2}=5.55 \mathrm{~Hz}$, respectively. That means twice of the resonant frequency of the vertical beam is very close to the resonant frequency of the horizontal beam. This closeness and the growth of the parametric resonance at this distance causes the higher branch of the softening behavior of the horizontal beam and the higher branch of the hardening behavior of vertical beam to overlap. At smaller distances 
below $30 \mathrm{~mm}$, under the effect of the hysteresis, this overlap part becomes unpredictable. Besides, if the distance is very small, the energy collected from the horizontal beam is low. Therefore, the distance cannot be set below $30 \mathrm{~mm}$. The optimal distance is around $34 \mathrm{~mm}$ where the energy from both beams has a relatively high level in a broader frequency bandwidth.

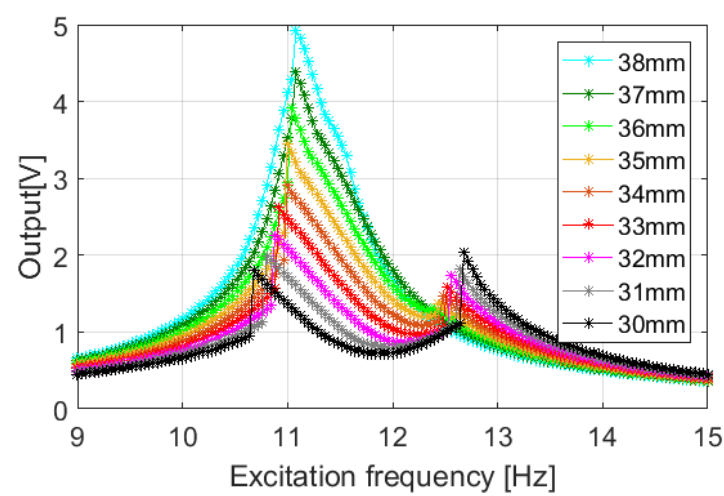

(a)

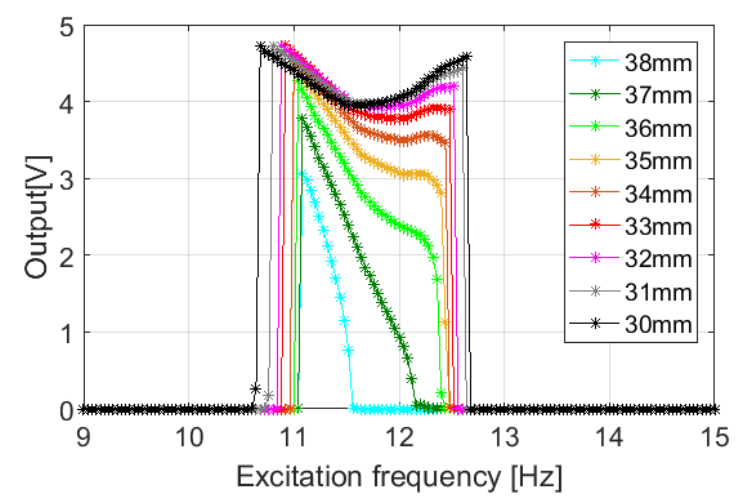

(b)

Figure 16: Simulated output voltage for different distance $d$ with base excitation of $0.5 g$ a) horizontal beam, b) vertical beam.

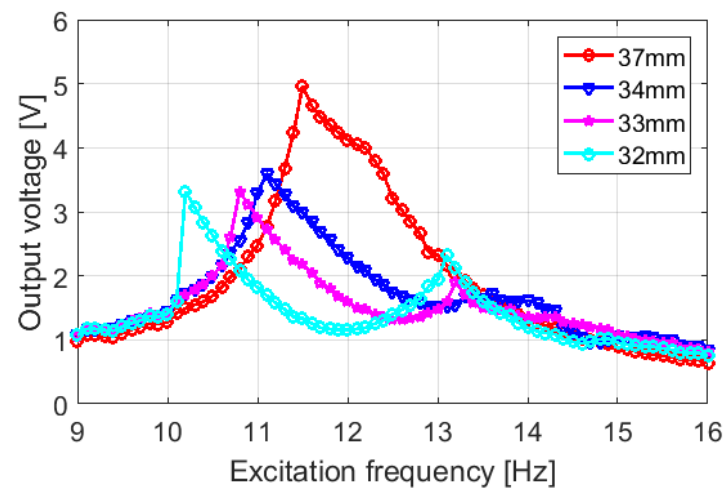

(a)

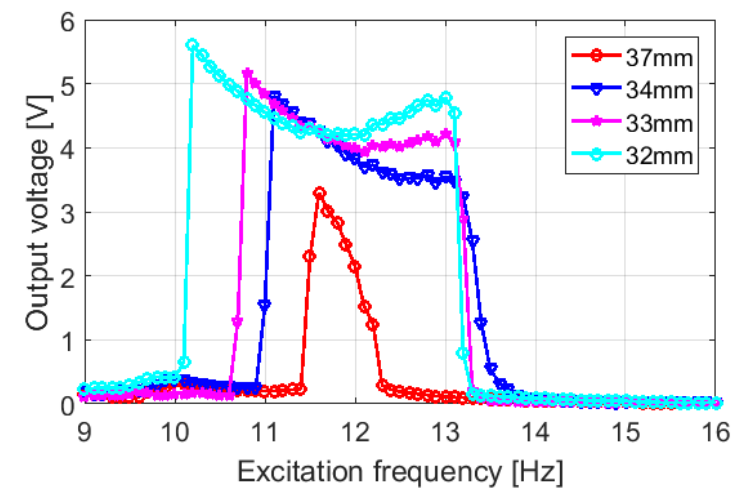

(b)

Figure 17: Experimental output voltage for different distance $d$ with base excitation of $0.5 \mathrm{~g}$ a) horizontal beam, b) vertical beam.

To validate the influence of the distance between the magnets on the frequency bandwidth, a series of experiments was conducted. Figures $17 \mathrm{a}$ and $17 \mathrm{~b}$ present the output voltage as the initial distance between magnets varies at the excitation level of $0.5 \mathrm{~g}$. As numerical simulations revealed, decreasing the distance between two magnets broadens the frequency bandwidth and boosts the parametric resonance response. On the contrary, the maximum output voltage generated by the horizontal beam decreases as the distance decreases. The optimal initial distance is around $d=33 \mathrm{~mm}$ which is almost the same as we conclude from the simulation. 
To examine the effect of the external resistance on the output power, the output power versus different load resistance is presented in Figure 18. The power is calculated according to $P=V^{2} / R$, where $R$ is the external load resistance and $V$ is the maximum output voltage in the frequency output. In Figure 18, two cases of $d=32 \mathrm{~mm}$ and $d=34 \mathrm{~mm}$ are studied. It can be observed that the optimal resistance for the vertical beam is $2.6 M \Omega$ and the optimal resistance for the horizontal beam is $1.2 \mathrm{M} \Omega$ regardless of the initial distance $d$ between two magnets. The output power from the vertical beam by parametric resonance is much greater than that from the horizontal beam by direct resonance. Therefore, the optimal load resistance is 2.6 M $\Omega$ as the tiny difference of the power from the horizontal beam can be neglected. Furthermore, when the distance is small $(d=32 \mathrm{~mm})$, the power of the parametric resonance increases. On the contrary, the power of direct resonance decreases. This is consistent with the output voltage frequency response presented in Figure 16a and $16 \mathrm{~b}$.

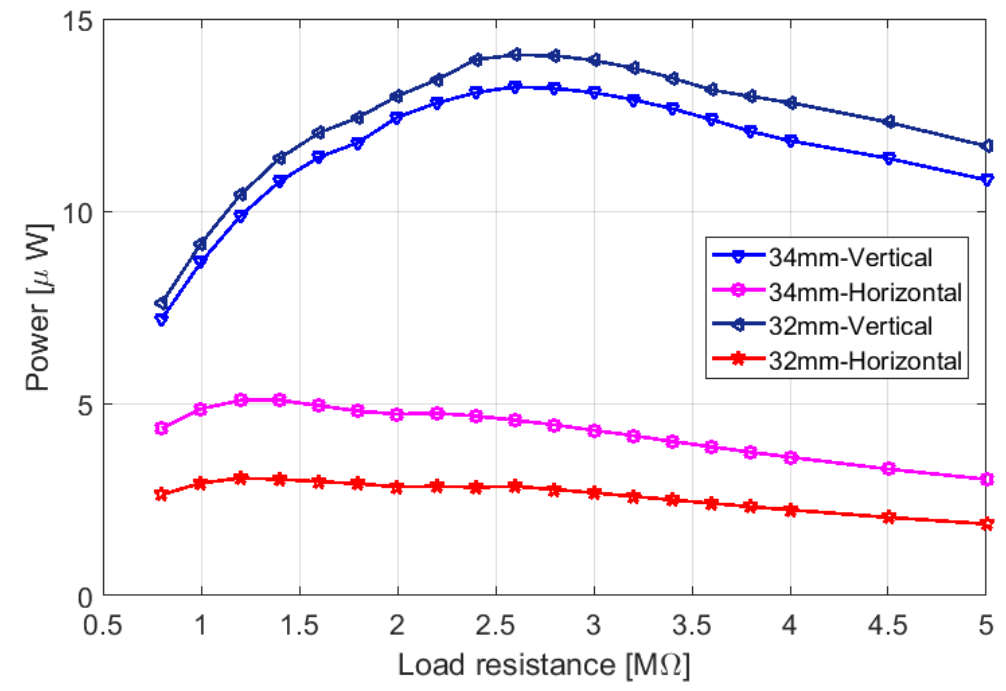

Figure 18: Simulation results of output power for different external load resistance.

\section{Conclusions}

A parametric resonator with low threshold excitation for vibration energy harvesting is proposed. The harvester comprises two piezoelectric cantilever beams, each with a magnetic tip. One is placed in the horizontal plane, the other in the vertical plane. The magnetic force is carefully tailored to decrease the threshold excitation level required to trigger parametric resonance. By controlling the initial distance between two magnets, the parametric resonance of the vertical beam is activated at a low excitation level and the frequency bandwidth of the energy harvester increases.

To simulate the response of the harvester, a two-degree-of-freedom mathematical model including magnetic interaction is developed. Four coupled differential equations are obtained to predict the dynamic behavior and output voltage of the energy harvester. The governing equations are solved numerically and analytically. Experiments were pursued to verify the 
mathematical model. Compared to a single piezoelectric cantilever beam, the initial threshold excitation level needed to trigger the parametric resonance decreases from $5.5 \mathrm{~g}$ to $0.3 \mathrm{~g}$ by the proposed design. Because of the combination of hardening and softening behavior, the parametric resonance of the vertical beam has a flattened and broader frequency bandwidth. Unlike traditional systems with cubic nonlinearity and parametric resonators, the harvester response is more robust to variation of initial conditions as the hysteresis region is minimized and the response lies on the high oscillation branch.

As the magnets have the key role in the system dynamics, the effects of the initial distance between magnets on the response are investigated. Considering the direct resonance of the horizontal beam, the optimal initial distance is found around $34 \mathrm{~mm}$ where both beams have a large output with broad frequency bandwidth. The optimal external load resistance is estimated to be 2.6 M . In summary, the direct resonance of the horizontal beam and the parametric resonance of the vertical beam are carefully merged to increase the efficiency of vibration energy harvesting by producing a large output over a wider frequency bandwidth.

\section{References}

\section{References}

[1] S. Priya, D. J. Inman, Energy harvesting technologies, Vol. 21, Springer, 2009.

[2] M. I. Younis, MEMS linear and nonlinear statics and dynamics, Vol. 20, Springer Science \& Business Media, 2011.

[3] F. Cottone, H. Vocca, L. Gammaitoni, Nonlinear energy harvesting, Physical Review Letters 102 (8) (2009) 080601.

[4] S. C. Stanton, C. C. McGehee, B. P. Mann, Nonlinear dynamics for broadband energy harvesting: Investigation of a bistable piezoelectric inertial generator, Physica D: Nonlinear Phenomena 239 (10) (2010) 640-653.

[5] A. Ibrahim, S. Towfighian, M. I. Younis, Dynamics of transition regime in bistable vibration energy harvesters, Journal of Vibration and Acoustics 139 (5) (2017) 051008.

[6] B. Ando, S. Baglio, C. Trigona, N. Dumas, L. Latorre, P. Nouet, Nonlinear mechanism in mems devices for energy harvesting applications, Journal of Micromechanics and Microengineering 20 (12) (2010) 125020.

[7] M. Ferrari, V. Ferrari, M. Guizzetti, B. Andò, S. Baglio, C. Trigona, Improved energy harvesting from wideband vibrations by nonlinear piezoelectric converters, Sensors and Actuators A: Physical 162 (2) (2010) 425-431.

[8] H. Vocca, I. Neri, F. Travasso, L. Gammaitoni, Kinetic energy harvesting with bistable oscillators, Applied Energy 97 (2012) 771-776.

[9] W. Yang, S. Towfighian, Performance of a bi-stable resonator with random input vibrations, in: ASME 2016 International Design Engineering Technical Conferences and Computers and Information in Engineering Conference, American Society of Mechanical Engineers, 2016, pp. V006T09A051-V006T09A051. 
[10] Y. Chen, T. E. Pollock, A. Salehian, Analysis of compliance effects on power generation of a nonlinear electromagnetic energy harvesting unit; theory and experiment, Smart Materials and Structures 22 (9) (2013) 094027.

[11] D. A. Barton, S. G. Burrow, L. R. Clare, Energy harvesting from vibrations with a nonlinear oscillator, Journal of Vibration and Acoustics 132 (2) (2010) 021009.

[12] S. P. Beeby, R. Torah, M. Tudor, P. Glynne-Jones, T. O'Donnell, C. Saha, S. Roy, A micro electromagnetic generator for vibration energy harvesting, Journal of Micromechanics and Microengineering 17 (7) (2007) 1257.

[13] J. Zhou, R. N. Miles, Sensing fluctuating airflow with spider silk, Proceedings of the National Academy of Sciences (2017) 201710559.

[14] H. Wu, L. Tang, Y. Yang, C. K. Soh, Development of a broadband nonlinear two-degreeof-freedom piezoelectric energy harvester, Journal of Intelligent Material Systems and Structures 25 (14) (2014) 1875-1889.

[15] L. Tang, Y. Yang, A nonlinear piezoelectric energy harvester with magnetic oscillator, Applied Physics Letters 101 (9) (2012) 094102.

[16] I. Sari, T. Balkan, H. Kulah, An electromagnetic micro power generator for wideband environmental vibrations, Sensors and Actuators A: Physical 145 (2008) 405-413.

[17] B. Andò, S. Baglio, F. Maiorca, C. Trigona, Analysis of two dimensional, wide-band, bistable vibration energy harvester, Sensors and Actuators A: Physical 202 (2013) 176182.

[18] L. Xiong, L. Tang, B. R. Mace, Internal resonance with commensurability induced by an auxiliary oscillator for broadband energy harvesting, Applied Physics Letters 108 (20) (2016) 203901.

[19] L.-Q. Chen, W.-A. Jiang, M. Panyam, M. F. Daqaq, A broadband internally resonant vibratory energy harvester, Journal of Vibration and Acoustics 138 (6) (2016) 061007.

[20] W. Yang, S. Towfighian, A hybrid nonlinear vibration energy harvester, Mechanical Systems and Signal Processing 90 (2017) 317-333.

[21] W. Yang, S. Towfighian, Internal resonance and low frequency vibration energy harvesting, Smart Materials and Structures 26 (9) (2017) 095008.

[22] M. F. Daqaq, C. Stabler, Y. Qaroush, T. Seuaciuc-Osório, Investigation of power harvesting via parametric excitations, Journal of Intelligent Material Systems and Structures 20 (5) (2009) 545-557.

[23] Y. Jia, J. Yan, K. Soga, A. A. Seshia, A parametrically excited vibration energy harvester, Journal of intelligent material systems and structures 25 (3) (2014) 278-289.

[24] Y. Jia, A. A. Seshia, An auto-parametrically excited vibration energy harvester, Sensors and Actuators A: Physical 220 (2014) 69-75. 
[25] M. A. Karami, J. R. Farmer, D. J. Inman, Parametrically excited nonlinear piezoelectric compact wind turbine, Renewable energy 50 (2013) 977-987.

[26] R. Rand, P. Holmes, Bifurcation of periodic motions in two weakly coupled van der pol oscillators, International Journal of Non-Linear Mechanics 15 (4-5) (1980) 387-399.

[27] A. H. Nayfeh, D. T. Mook, Nonlinear oscillations, John Wiley \& Sons, 2008. 